\title{
Simultaneous analysis of four stereoisomers of anti-benzo $[a]$ pyrene diol epoxide-deoxyguanosine adducts in short oligodeoxynucleotides using reversed-phase high-performance liquid chromatography
}

\author{
Feng Feng, Hailin Wang* \\ State Key laboratory of Environmental Chemistry and Ecotoxicology, Research Center for Eco-Environmental Sciences, \\ Chinese Academy of Sciences, Beijing 100085, China
}

Available online 5 May 2007

\begin{abstract}
anti-Benzo[ $[a]$ pyrene-7,8-dihydrodiol-9,10-epoxide (anti-BPDE), a reactive metabolite of the environmental carcinogen benzo[a]pyrene, predominantly binds to deoxyguanine in DNA and forms four stereoisomeric adducts. Here we developed an improved method for simultaneous analysis and purification of four stereoisomeric adducts in short oligonucleotides using reversed-phase high-performance liquid chromatography, providing a selection strategy of stationary phase for analysis and separation of polyaromatic hydrocarbon-DNA adducts. This work demonstrates that secondary retention of oligonucleotides on $\mathrm{C} 18$ stationary phases induced by exposed silanol heavily affects the separation of four stereosiomeric adducts on C18 stationary phases, and the silicone polymer monolayer coating for completely capping exposed silica or silanol greatly reduces such secondary retention, thereby displaying a much better resolution of the four stereoisomeric adducts. We further demonstrate that aromatic group (phenyl)-based stationary phase can significantly improve stereoisomeric separation of four anti-BPDE-deoxyguanosine $(\mathrm{dG})$ adducts in short oligonucleotides over nonaromatic C18 stationary phase due to enhancement of the selective interaction with aromatic anti-BPDE moiety in oligonucleotides. The developed method was also used for purification and preparation of anti-BPDE-oligoncleotide adducts.
\end{abstract}

(C) 2007 Elsevier B.V. All rights reserved.

Keywords: Benzo[a]pyrene; ( \pm -anti-BPDE; DNA adducts

\section{Introduction}

Benzo $[a]$ pyrene is one of the polycyclic aromatic hydrocarbons (PAHs) found in coal tar, tobacco smoke, broiled foods, automotive exhausts, forest fires, and other polluted environments [1]. Since its detection in coal tar as one of the principal carcinogens [2], benzo[ $a$ ] pyrene is often chosen as an important model chemical carcinogen for investigating the mutagenic and carcinogenic properties of PAHs [3]. Benzo[ $a$ ]pyrene is not biologically active per se, but requires metabolic activation to elicit its hazardous properties. The most widely accepted metabolic activation pathway involves three steps: (I) formation of epoxide at the 7,8-position of benzo[ $a]$ pyrene by action of CYP $1 \mathrm{~A} 1$; (II) formation of 7,8-dihydrodiol from benzo[ $[a]$ pyrene epoxide catalyzed by epoxide hydrolase; and (III) formation of an epox-

\footnotetext{
* Corresponding author. Tel.: +86 1062849600 ; fax: +86 1062849667 . E-mail address: hlwang@rcees.ac.cn (H. Wang).
}

ide in the bay region at the 9,10-position of the benzo[ $a]$ pyrene 7,8-dihydrodiol by a second attack by CYP1A1, yielding the ultimate carcinogen (+)-anti-BPDE [4-6]. Along this metabolic pathway, $( \pm)$-syn-BPDE and (-)-anti-BPDE are also probably generated [7-9]. Racemic anti-BPDE is reported to be more mutagenic than the diastereomer $( \pm$-syn-BPDE [10-12], and the mutagenic potencies of the two anti-BPDE enantiomers are different in selected bacterial and mammalian cell systems. The $(+)$-anti-BPDE isomer is tumorigenic on mouse skin and in the lungs of new born mice [13,14], while the (-)-anti-BPDE enantiomer is not. In the TA100 strain, as well as in mammalian Chinese hamster ovary cells (V79 cells), the activity of the $(+)$-isomer is 4-6 times greater than that of (-)-anti-BPDE $[15,16]$. But in two separated genes (hisG and gpt loci) from S. typhimurium TA100, the mutagenic efficiency (mutation frequency per DNA lesion) of (-)-anti-BPDE is higher than that of the (+)-enantiomer [17]. From the view of molecular biology at molecular levels, these biological differences may be associated with the underlined conformations of stereoisomers of 


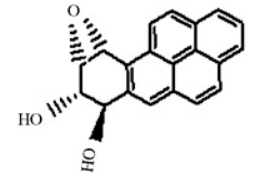

(+)-anti-BPDE

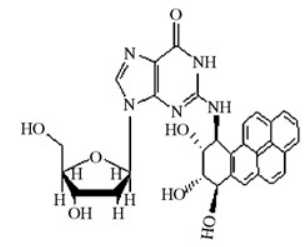

trans-(+)-anti-BPDE- $N^{2}-\mathrm{dG}$

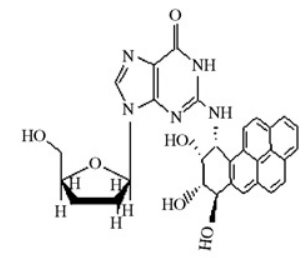

cis-(+)-anti-BPDE- $N^{2}-\mathrm{dG}$

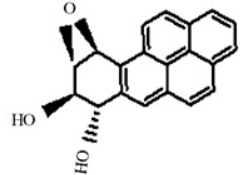

(-)-anti-BPDE
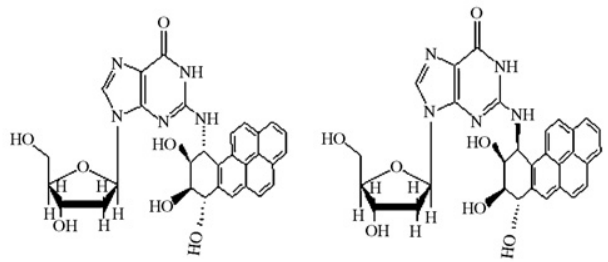

trans-(-)-anti-BPDE- $N^{2}-\mathrm{dG} \quad$ cis-(-)-anti-BPDE- $N^{2}-\mathrm{dG}$

Fig. 1. Structures of ( \pm )-anti-BPDE enantiomer (top) and the moieties of four BPDE- $N^{2}-$ dG stereoisomers in oligonucleotides (bottom).

anti-BPDE-DNA adducts (Fig. 1) resulting from covalent binding of BPDE to DNA. Therefore, the synthesis and analysis of the stereoisomers of the DNA adducts are very important to understand their biological effects.

Here we developed an improved HPLC method for simultaneous analysis and preparation of four stereoisomers of anti-BPDE-deoxyguanosine $(\mathrm{dG})$ adducts in an oligodeoxynucleotide of $16 \mathrm{mer}$. Previously, Shibutani et al. and Cheng et al. used (+)-anti-BPDE and (-)-anti-BPDE to synthesize and separate their corresponding trans- and cis- BPDE-dG adducts $[18,19]$, respectively. However, the two isolated anti-BPDE isomers are difficult to prepare because of their instability in liquid phase. In contrast, racemic anti-BPDE is relatively easy to be synthesized and purified. In this work, we directly used racemic anti-BPDE to synthesize the anti-BPDE-dG adducts in the 16 mer followed by simultaneous analysis and preparation of four stereoisomeric adducts. Furthermore, we compared the retention and separation of BPDE-oligonucleotide adducts on four reversed-phase stationary phases. It was found that the elimination of secondary retention of oligonucleotides and the enhancement of the selective interaction between BPDE adduct moieties and immobilized ligands may improve the stereoisomeric separation of BPDE-oligonucleotide adducts. This may reveal a mechanism-based rule for developing new stationary phases for analysis and separation of DNA adducts. The prepared stereoisomers of BPDE-16mer can be further used for synthesis of DNA adduct probes with specific conformations to study the DNA repair, transcription, replication, and mutation, and to monitor human exposure of carcinogenic PAHs.

\section{Materials and methods}

\subsection{Materials}

Caution: BPDE is carcinogenic. Appropriate care should be exercised when handling BPDE.

( \pm )-r-7,t-8-Dihydroxy- $t$-9,10-epoxy-7,8,9,10-tetrahydrobenzo[a]pyrene (anti) $[( \pm)$-anti-BPDE] was gift from Dr. Chris
Le at the University of Alberta (supplied by the National Cancer Institute Chemical Carcinogen Reference Standard Repository, Midwest Research Institute, Kansas City, MO,USA). The unmodified oligonucleotide of $5^{\prime}$-CCC ATT ATG CAT AAC C-3' (16mer) were synthesized by TaKaRa Biotech (Dalian, China). The purity of the 16 mer was checked by HPLC prior to use. Dimethyl sulfoxide (DMSO) and triethylamine used for the synthesis of damaged oligodeoxynucleotides were of analytical grade and supplied by Sigma-Aldrich (St. Louis, MO, USA). Methanol was purchased from Fisher (Pittsburgh, PA, USA) and was of HPLC grade. Other chemicals and solvents used were of analytical or HPLC grade.

\subsection{Instrumentation}

HPLC analyses of the synthetic BPDE-DNA adducts were conducted on a Hitachi L-2000 series liquid chromatography system from Hitachi (Tokyo, Japan). The Hitachi L-2000 HPLC system consisted of a model L-2130 low pressure gradient pump and a model Hitachi L-2200 autosampler with an injection volume from 0 to $100 \mu \mathrm{L}$, equipped with the first detector of Hitachi L-2455 (1024 bits) diode array spectrophotometric detector and the second detector of Hitachi L-2485 fluorescence detector. These detectors were connected to and controlled by a Hitachi EZChrom Elite chromatography data system.

\subsection{HPLC columns}

During our experiments, four analytical HPLC columns were used. The first is a venusil XBP C18 column $(250 \mathrm{~mm} \times 4.6 \mathrm{~mm}$ I.D., $5 \mu \mathrm{m}$ particle size, $100 \AA$ pore size, $99.999 \%$ silica purity, metal impurities $<10 \mu \mathrm{g} / \mathrm{g}$, column 1), the second is a Capcell Pak C18 MG column $(250 \mathrm{~mm} \times 4.6 \mathrm{~mm}$ I.D., $5 \mu \mathrm{m}$ particle size, $100 \AA$ pore size, metal impurities $<5 \mu \mathrm{g} / \mathrm{g}$, column 2), the third is a Capcell Pak C18 SG column $(150 \mathrm{~mm} \times 4.6 \mathrm{~mm}$ I.D., $5 \mu \mathrm{m}$ particle size, $300 \AA$ pore size, metal impurities $<5 \mu \mathrm{g} / \mathrm{g}$, column 3), and the last is a Capcell Pak Ph UG column $(250 \mathrm{~mm} \times 4.6 \mathrm{~mm}$ I.D., $5 \mu \mathrm{m}$ particle size, $120 \AA$ pore size, the metal impurities $<5 \mu \mathrm{g} / \mathrm{g}$, column 4 ). Column 1 was purchased 
from Agela Technologies (DE, USA) and columns 2, 3, and 4 were purchased from Shiseido (Tokyo, Japan).

\subsection{Synthesis of damaged BPDE-DNA adduct}

The $16 \mathrm{mer}$ of $5^{\prime}$-CCCATTATGCATAACC-3' and racemic anti-BPDE were used to synthesize BPDE-DNA adduct in vitro according to the procedure previously described by Carnelley et al. [20], with slight modifications. In brief, the $16 \mathrm{mer}$ was diluted in $20 \mathrm{mM}$ phosphate buffer $(\mathrm{pH} \mathrm{11)}$, containing $0.9 \%$ triethylamine, to a concentration of $60 \mu \mathrm{M}$ in a volume of $400 \mu \mathrm{L}$. A solution of $3 \mathrm{mM}( \pm)$-anti-BPDE dissolved in anhydrous DMSO was freshly prepared, and $40 \mu \mathrm{L}$ was added to the oligonucleotide solution. This is corresponding to a BPDE:oligonucleotide ratio of 5:1 [21]. The reaction was carried out at room temperature over $24 \mathrm{~h}$ in the dark.

\subsection{Analysis of BPDE-DNA adduct}

The reaction products were initially assessed on the analytical column using a linear gradient elution and the elution procedure for each column was individually optimized. The elution of the products was monitored in series by diode array detection (DAD) (absorbance wavelength 200-400 nm) and fluorescence detection (excitation wavelength $=343 \mathrm{~nm}$, emission wavelength $=400 \mathrm{~nm}$ for BPDE). Two mobile-phase solutions (solvents $\mathrm{A}$ and $\mathrm{B}$ ) were prepared. The solvent $\mathrm{A}$ consisted of $10 \mathrm{mM}$ acetate buffer $(\mathrm{pH}$ 6.9), $0.14 \%$ triethylammonium and $6 \%$ methanol, and the solvent $\mathrm{B}$ consisted of $10 \mathrm{mM}$ acetate buffer $(\mathrm{pH}$ 6.9), $0.14 \%$ triethylammonium and $80 \%$ methanol. The HPLC analysis was carried out at room temperature. In order to obtain the best separation efficiency, elution methods for each column were optimized.

For column 1, the samples were eluted at a flow-rate of $0.9 \mathrm{~mL} / \mathrm{min}$. For the first $5 \mathrm{~min}$, an isocratic elution with a mobile phase of $21.6 \%$ solvent B and $78.4 \%$ solvent A was used; then a gradient elution from $21.6 \%$ to $35.1 \%$ solvent B for the next $25 \mathrm{~min}$ was used; followed by a $10 \mathrm{~min}$ isocratic $35.1 \%$ solvent $\mathrm{B}$; and finally a $100 \%$ solvent $\mathrm{B}$ was used for elution over 15 min.

For column 2, the samples were eluted at a flow-rate of $0.75 \mathrm{~mL} / \mathrm{min}$. For the first $5 \mathrm{~min}$, an isocratic elution with a mobile phase of $21.6 \%$ solvent B and $78.4 \%$ solvent A was used; then a gradient elution of solvent B increased from $21.6 \%$ to $35.1 \%$ for the next $25 \mathrm{~min}$, followed by a $6 \mathrm{~min}$ isocratic elution of $35.1 \%$ solvent B; and finally a $100 \%$ solvent B was used for elution over $15 \mathrm{~min}$. For column 3, the same gradient elution procedure was used as for column 2.

For column 4, the samples were eluted at the flow-rate of $0.75 \mathrm{~mL} / \mathrm{min}$, too. For the first $5 \mathrm{~min}$, an isocratic condition with a mobile phase consisting of $14.9 \%$ solvent $B$ and $85.1 \%$ solvent A was used; then a gradient elution of solvent B increased from $14.9 \%$ to $35.1 \%$ for the next 20 min was used, followed by a 5 min isocratic elution of $35.1 \%$ solvent B; and finally a $100 \%$ of solvent B elution was adopted over $15 \mathrm{~min}$.

\subsection{Purification of single stereoisomer of BPDE-DNA adduct}

The columns 2 and 4 were chosen to purify single stereoisomers of anti-BPDE-16mer adduct because of their better resolution during HPLC analysis. The elution conditions were used as those for HPLC analysis of four stereoisomers of antiBPDE-16mer adduct. The injection volumes were increased up to $99 \mu \mathrm{L}$ for preparative separation. After the single stereoisomers of the adducts were collected, the collected fractions were dried using lyophilization, then stored in refrigerator at $-20{ }^{\circ} \mathrm{C}$.

\subsection{Identification of anti-BPDE-DNA adducts stereoisomers}

The stereoisomers of the anti-BPDE-16mer adducts were identified based on yield, relative retention strength, UV spectrum (200-400 nm) according to the work of Shibutani et al. and Cheng et al. [18,19]. In their work, the identities of the adduct stereoisomers were examined using HPLC analysis (C18 column), NMR, and circular dichroism. Based on their work, we can identify trans or cis stereoisomers on C18 column. On C18 column, the four stereoisomeric adducts were eluted out in the order of cis-anti-BPDE-16mer A, trans-anti- BPDE-16mer A, trans-anti-BPDE-16mer B, and cis-anti-BPDE-16mer B. However, on the Ph column the elution order of adducts has not been reported, so we may use the purified single stereoisomers of antiBPDE-16mer adducts from $\mathrm{C} 18$ column to identify the peaks on Ph column.

\section{Results and discussion}

\subsection{Silicone polymer monolayer coated silica $\mathrm{Cl} 18$ for analysis of BPDE-DNA adduct}

The reactive metabolite $( \pm)$-anti-BPDE can react with $\mathrm{dG}$ in the designed 16mer oligonucleotide, which only contains a single $\mathrm{dG}$, and yields four anti-BPDE- $N^{2}-\mathrm{dG}$ adducts (Fig. 1). In the reaction solution, a mixture of unreacted $16 \mathrm{mer}$ oligonucleotide, BPDE- $N^{2}-\mathrm{dG}-16$ mer adducts, and numerous BPDE hydrolysis products was present. These ingredients were first separated by reversed-phase HPLC using silica-based C18 stationary phases (columns 1 and 2). For the preliminary separation of the product mixture, a linear 0-90\% methanol gradient was employed. Under such conditions, unreacted $16 \mathrm{mer}$ was eluted out first, followed by the BPDE- $-N^{2}-\mathrm{dG}-16$ mer adducts, and the BPDE hydrolysis products were eluted out last (data not shown). The unreacted $16 \mathrm{mer}$ has a strong absorbance at $260 \mathrm{~nm}$ but no fluorescence response (excitation wavelength $=343 \mathrm{~nm}$, emission wavelength $=400 \mathrm{~nm}$ ) as typical DNA (Fig. 2). The BPDE- $N^{2}-$ dG-16mer adducts have an additive absorbance spectrum of BPDE and DNA, and also have fluorescence response of BPDE moiety (Fig. 2). The BPDE hydrolysis products have much stronger fluorescence signals but poorer UV $260 \mathrm{~nm}$ absorbance than anti-BPDE- $N^{2}-\mathrm{dG}$ adducts at the same concentration. In following work, we focused on the 


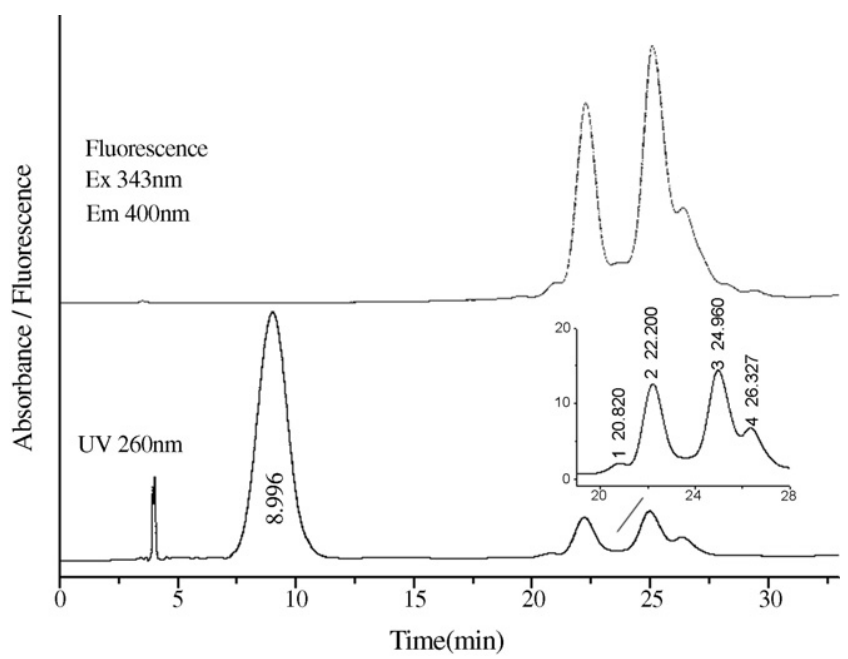

Fig. 2. Chromatograms obtained from HPLC analysis of the reaction products of the ( \pm )-anti-BPDE and 16mer using a Capcell Pak C18 MG column (column $2)$. The analytes were simultaneously monitored by DAD (200-400 nm) and fluorescence (Ex $343 \mathrm{~nm}, \mathrm{Em} 400 \mathrm{~nm}$ ). The eluted species including unmodified DNA (8.996 $\mathrm{min})$ and four stereoisomers of anti-BPDE-16mer $(20.820,22.200$, 24.960 , and 26.327 min). Peaks $1,2,3$, and 4 represent $c i s$-anti-BPDE- $N^{2}-\mathrm{dG}$ 16 mer A, trans-anti-BPDE- $N^{2}-\mathrm{dG}-16$ mer A, trans-anti-BPDE- $N^{2}-\mathrm{dG}-16 \mathrm{mer}$ $\mathrm{B}$, and cis-anti-BPDE- $N^{2}-\mathrm{dG}-16$ mer B. For the conditions of HPLC analysis see Section 2.

separation of four stereoisomers of anti-BPDE- $N^{2}-\mathrm{dG}-16 \mathrm{mer}$ adducts.

In order to simultaneously separate four stereoisomeric adducts of anti-BPDE- $N^{2}-\mathrm{dG}-16$ mer, a unique C18 stationary phase having a silicone monolayer coating on silica and high carbon content of $15 \%$ (column 2) was chosen because of the possible reduction of secondary retention of DNA with silanol or silica. Fig. 2 shows the chromatograms obtained from HPLC analysis of reaction products of antiBPDE and 16mer using column 2. In Fig. 2, dashed trace represents the fluorescence signal, and the solid trace represents the UV absorbance signal. By using column 2, the anti-BPDE-16mer adducts were separated into four peaks with retention times of 20.82, 22.20, 24.96 and $26.33 \mathrm{~min}$, which may be corresponding to $c i s-(+)$-anti-BPDE- $N^{2}-\mathrm{dG}-$ $16 \mathrm{mer}$, trans-(-)-anti-BPDE- $N^{2}-\mathrm{dG}-16 \mathrm{mer}$, trans-(+)-antiBPDE- $N^{2}-\mathrm{dG}-16 \mathrm{mer}$, and $c i s-(-)$ ant $i-\mathrm{BPDE}-N^{2}-\mathrm{dG}-16 \mathrm{mer}$. The four stereoisomers of the adducts were identified based on the literature of Shibutani et al. and Cheng et al. [18,19]. Cheng et al. employed (+)-anti-BPDE enantiomer and (-)-anti-BPDE enantiomer to synthesize BPDE-DNA adduct, respectively, and utilized circular dichroism spectra as well as NMR spectra to identify the four adducts. Similar work of synthesis and identification BPDE-DNA adducts has also been done by Shubutani et al. In Shibutani et al.'s work, the elution of (+)-anti-BPDE product was in the order of (+)-cis-BPDE-18mer (24.20 min) and (+)-trans-BPDE-18mer (25.3 min), respectively. Meanwhile, the (-)-trans-BPDE-18mer and (-)-cis-BPDE-18mer eluted at 24.5 and $25.3 \mathrm{~min}$, respectively [18]. In our work, the relative yield of the four stereoisomers of anti-BPDE- $N^{2}-\mathrm{dG}$ adducts also support this assignment of the peaks. According to peak identification by retention strength on $\mathrm{C} 18$ station- ary phases, the third adduct peak $(24.96 \mathrm{~min})$ is assigned as trans-(+)-anti-BPDE- $N^{2}-\mathrm{dG}$. In Fig. 2, this peak is the largest adduct peak $(\sim 44.3 \%$ of total UV peak area of all four adducts), consistent with the reaction property of anti-BPDE and DNA, which leads to predominant formation of transanti-BPDE- $N^{2}-\mathrm{dG}$ adduct (peaks 2 and 3 ) and to higher yield of trans-(+)-anti-BPDE- $N^{2}-\mathrm{dG}(\sim 44.3 \%)$ than trans-(-)-antiBPDE- $N^{2}-\mathrm{dG}$ (peak 2) $(\sim 36.7 \%)$ [19]. It is also reasonable that the adduct isomer cis-(+)-anti-BPDE- $N^{2}-\mathrm{dG}$ (peak 1) is very unstable [19] and gives smallest peak (peak 1) $(\sim 2.8 \%)$. However, further confirmation and validation for the optical configuration of each stereoisomer is needed and will be conducted using enzymatic hydrolysis combined with mass spectrometry, CD, and NMR in a separate work. In this work, we suggest that the peaks 1, 2, 3, and 4 in Fig. 2 are corresponding to cis-anti-BPDE- $N^{2}-\mathrm{dG}-16$ mer A, transanti-BPDE- $N^{2}-\mathrm{dG}-16$ mer A, trans-anti-BPDE- $N^{2}-\mathrm{dG}-16 \mathrm{mer}$ $\mathrm{B}$, and $c i$-anti-BPDE- $N^{2}-\mathrm{dG}-16$ mer B without illustration of specific optic configuration.

To achieve fast and efficient separation of four stereoisomers of anti-BPDE-16mer adducts, an optimized elution procedure (see experimental section) was chosen. Under such conditions, the cis-anti-BPDE- $N^{2}-\mathrm{dG}-16$ mer A, trans-anti-BPDE- $N^{2}-\mathrm{dG}$ 16mer A, and trans-anti-BPDE- $N^{2}-\mathrm{dG}-16$ mer B (peaks 1, 2, and 3) were well separated with a resolution of $R_{1,2}=0.71$, $R_{2,3}=1.15$ ), The peaks of trans-anti-BPDE- $N^{2}-\mathrm{dG}-16$ mer B and cis-anti-BPDE- $N^{2}-\mathrm{dG}-16$ mer B were partially separated with a resolution of $R_{3,4}=0.55$ ). In Fig. 2, the separation of the adduct isomers recorded by fluorescence signal is worse than that recorded by UV $260 \mathrm{~nm}$. This is due to more void volume involved in second detection (fluorescence detector).

To understand the factors governing separation for antiBPDE-ssDNA adduct isomers and further improve the separation of the adduct isomers, we further investigated the separation of anti-BPDE-16mer adducts on an uncoated silica based C18 stationary phase (column 1). It is evident that the separation of four stereoisomers of BPDE-16mer adducts became worse using the uncoated silica based C18 stationary phase (Fig. 3). In Fig. 3, three major peaks were eluted from the column with retention times of 19.1, 27.2, and $29.3 \mathrm{~min}$, corresponding to unmodified 16mer DNA (19.1 min) and two major BPDE-16mer stereoisomers (27.2 and $29.3 \mathrm{~min}$ ) which overlap with the other two minor stereoisomers (as shown below in Fig. 4). Under the optimized elution conditions for column 1, four stereoisomers of the BPDE-16mer adduct formed in reaction mixture were only separated into two peaks with a poor resolution of 0.38 . The two peaks were individually collected, and HPLC analysis using column 2 followed. It was found that either of the peaks could be separated into 2 or 3 peaks on column 2 (Fig. 4), confirming the poor separation of four adduct stereoisomers on column 1. In comparison with silicone polymer monolayer coated silicabased C18 stationary phase (Fig. 2), the poor separation of adduct stereoisomers on uncoated silica based C18 stationary phase (Fig. 3) suggests that the reduction of silanol exposure can effectively improve the separation of anti-BPDE-DNA adducts. 


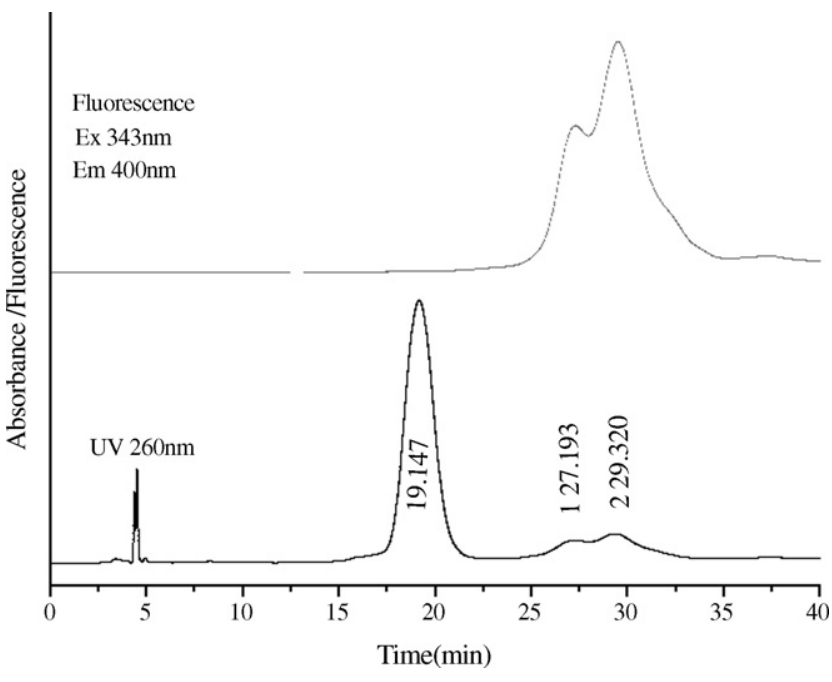

Fig. 3. HPLC analysis of the reaction products of the ( \pm -anti-BPDE and unmodified 16mer oligonucleotide using the uncoated ODS column (column 1). The analytes were monitored by DAD and fluorescence detection as described in Fig. 2. Peaks 1 and 2 are predominantly trans-anti-BPDE- $-N^{2}-\mathrm{dG}-16$ mer A and trans-anti-BPDE- $N^{2}-\mathrm{dG}-16 m e r \mathrm{~B}$, respectively. For the HPLC conditions see Section 2.

\subsection{Effect of pore size on separation}

In addition to polymer coating on silica, we investigated the effect of pore size on the separation of anti-BPDE- $N^{2}-\mathrm{dG}$ 16 mer adducts. Fig. 5 shows the separation of reaction mixture of anti-BPDE and 16mer using a stationary phase with a large pore size (column 3 ). The used stationary phase also has silicone polymer monolayer coating on silica and immobilized C18 group, which is the same as the column 2, but has much larger pore size $(300 \AA)$. For stationary phases packed in columns 1 and 2 , the particle pore sizes are only about $100 \AA$. It is evident

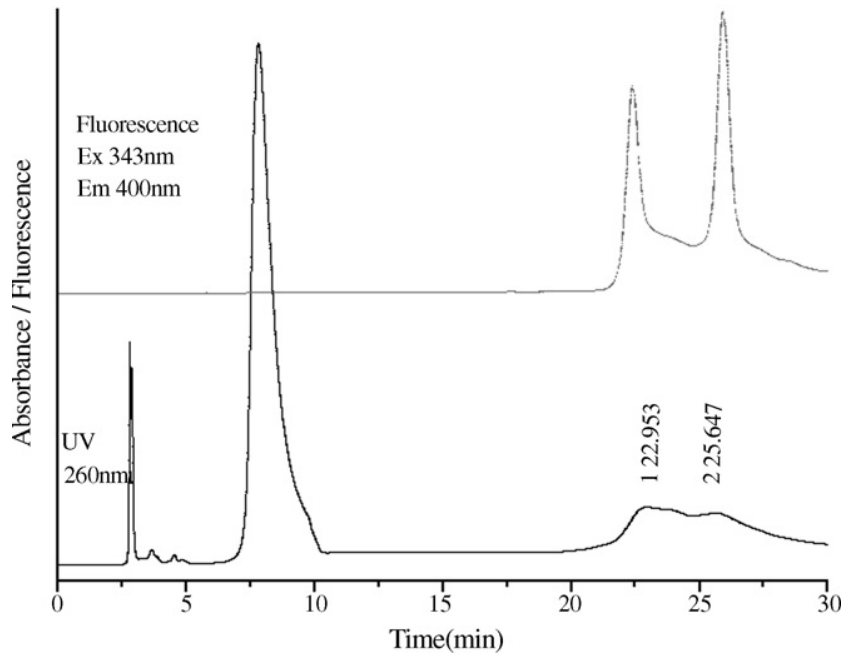

Fig. 5. HPLC analysis of the reaction products of the ( \pm -anti-BPDE and unmodified 16mer oligonucleotide using a Capcell Pak C18 SG column $(300 \AA$ pore size) showing peak tailing of DNA and DNA adducts. The analytes were monitored by DAD (200-400 nm) and fluorescence detection. For the HPLC conditions see Section 2.

that large pore size cannot effectively improve the separation of anti-BPDE-16mer adducts due to significant peak tailing (Fig. 5).

\subsection{Analysis of anti-BPDE-16mer using phenyl silica stationary phase}

As described above, capping of silanol by silicone polymer monolayer coating can significantly reduce the secondary retention of oligonucleotide, and thereby improve the separation of anti-BPDE adduct stereoisomers. To further improve the separation, we investigate the separation on $\mathrm{Ph}$ (phenyl) sta-

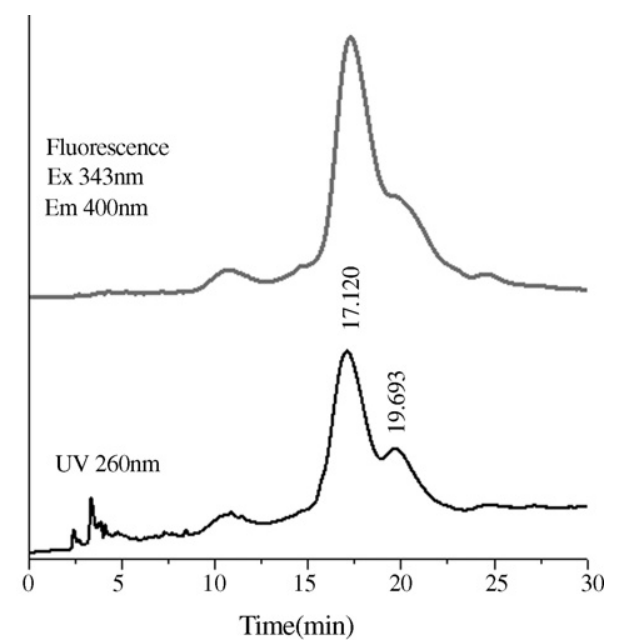

Fig. 4. HPLC analysis of fraction 1 (left) and fraction 2 (right) using Capcell Pak C18 MG column (column 2). The fractions 1 and 2 were collected from HPLC preparation using the uncoated ODS column (column 1) as shown in Fig. 3. For fraction 1, an isocratic elution with a mobile phase of $25.7 \%$ solvent B and $74.3 \%$ solvent A was used for the first $5 \mathrm{~min}$; then a gradient elution of solvent B increased from $25.7 \%$ to $35.1 \%$ for the next 20 min. For fraction 2 , an isocratic elution with a mobile phase of $27 \%$ solvent B and $73 \%$ solvent A was used for the first $5 \mathrm{~min}$; then a gradient elution of solvent B increased from $27 \%$ to $35.1 \%$ for the next $25 \mathrm{~min}$. Under such conditions, the fraction 1 can be separated into two peaks (14.52 and $16.37 \mathrm{~min}$ ), and fraction 2 was separated into two peaks (17.12 and $19.70 \mathrm{~min})$. The analytes were monitored by both DAD $(200-400 \mathrm{~nm})$ and fluorescence detection (Ex: $343 \mathrm{~nm}$; Em: $400 \mathrm{~nm})$. For other HPLC conditions see Section 2. 


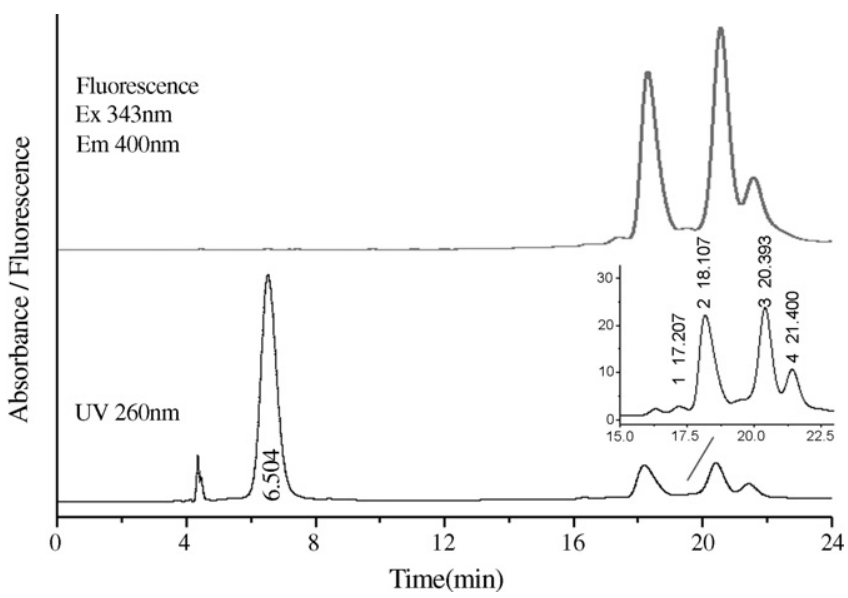

Fig. 6. Chromatograms obtained from HPLC analysis of the reaction products of the ( \pm )-anti-BPDE and 16mer using a Capcell Pak Ph UG column (column 4). The analytes were simultaneously monitored by DAD $(200-400 \mathrm{~nm})$ and fluorescence (Ex: $343 \mathrm{~nm}$; Em: $400 \mathrm{~nm}$ ). Eluted species include unmodified DNA (6.51 min) and four stereoisomers of anti-BPDE-16mer (17.21, 18.11, 20.40, and $21.40 \mathrm{~min}$ ). Peaks $1,2,3$, and 4 are cis-anti-BPDE- $N^{2}-\mathrm{dG}-16 \mathrm{mer}$ $\mathrm{A}$, trans-anti-BPDE- $-N^{2}-\mathrm{dG}-16 \mathrm{mer} \mathrm{A}$, trans-anti-BPDE- $-N^{2}-\mathrm{dG}-16 \mathrm{mer} \mathrm{B}$, and cis-anti-BPDE- $N^{2}-\mathrm{dG}-16 \mathrm{mer}$ B, respectively. For the conditions for HPLC analysis see Section 2.

tionary phase (column 4). The chosen Ph stationary phase also has silicone polymer monolayer coating on silica but has functional group of phenyl instead of $\mathrm{C} 18$. The functional group of phenyl has aromaticity, and it is expected that it should have better selective interaction with aromatic anti-BPDE moiety in oligonucleotide adducts in comparison with bases and backbone of oligonucleotides. Fig. 6 shows chromatographic analysis of four stereoisomers using the $\mathrm{Ph}$ stationary phase. In Fig. 6, there are four anti-BPDE-16mer adducts observed, which have both UV 260 absorbance and strong fluorescence response (Ex $343 \mathrm{~nm}, \mathrm{Em} 400 \mathrm{~nm}$ ). These peaks were identified using known single adduct stereoisomers purified by column 2. The adduct stereoisomers were eluted out in the order of cis-anti-BPDE- $N^{2}-\mathrm{dG}-16 \mathrm{mer} \mathrm{A}(17.2 \mathrm{~min})$, trans-anti-
BPDE- $N^{2}-\mathrm{dG}-16 \mathrm{mer}$ A (18.1 min), trans-anti-BPDE- $N^{2}-\mathrm{dG}-$ 16 mer B (20.4 min), and cis-anti-BPDE- $N^{2}-\mathrm{dG}-16 \mathrm{mer}$ B (21.4 min). Compared with the separation on the C18 stationary phase (column 2, Fig. 2), it is evident that better separation of four adduct stereoisomers is achieved. The better separation may be achieved as expected from selective aromatic interaction. Indeed, the interaction between the unmodified 16mer (without strong aromaticity) and the phenyl groups on the stationary phase (column 4) greatly reduces, and the unmodified $16 \mathrm{mer}$ is eluted out by $18 \%$ methanol with a shorter retention $(6.5 \mathrm{~min})$. In contrast, the unmodified $16 \mathrm{mer}$ is eluted out by $22 \%$ methanol with longer retention time $(9.0 \mathrm{~min})$ on the $\mathrm{C} 18$ stationary phase (column 2). However, the stereoisomers of BPDE- $N^{2}-\mathrm{dG}-16 \mathrm{mers}$ are eluted by similar elution strength (24-28\% methanol) on columns 2 and 4 . These results may indicate the occurrence of $\pi-\pi$ interaction between the aromatic BPDE moiety of the adduct and the phenyl groups on the stationary phase (column 4). The peaks of cis-anti-BPDE- $N^{2}-\mathrm{dG}-16 \mathrm{mer} \mathrm{A}(18.1 \mathrm{~min})$ and trans-anti-BPDE- $N^{2}-\mathrm{dG}-16 \mathrm{mer}$ A (20.4 min) can be completely separated. The peaks of trans-anti-BPDE- $-N^{2}-\mathrm{dG}-16 \mathrm{mer}$ B (20.4 min) and cis-anti-BPDE- $N^{2}-\mathrm{dG}-16 \mathrm{mer}$ B $(21.4 \mathrm{~min})$ can be well separated with a resolution of 0.67 .

In addition to better separation achieved using phenyl stationary phase, the analytical speed also improved. One analysis can be done within $23 \mathrm{~min}$. Thus, faster analysis is another advantage of phenyl stationary phase (column 4).

\subsection{Purification of single stereoisomer of BPDE-16mer adduct}

The preparation of short BPDE-oligonucleotide adduct is usually involved with the amount of DNA of less than $10 \mu \mathrm{g}$. This can be satisfied using an analytical column. In this work, we purify the adduct stereoisomers using an analytical column. The purification was carried out using silicone polymer monolayer coated silica-based $\mathrm{C} 18$ and $\mathrm{Ph}$ stationary phases (columns 2 and 4), respectively. The injection volume increased
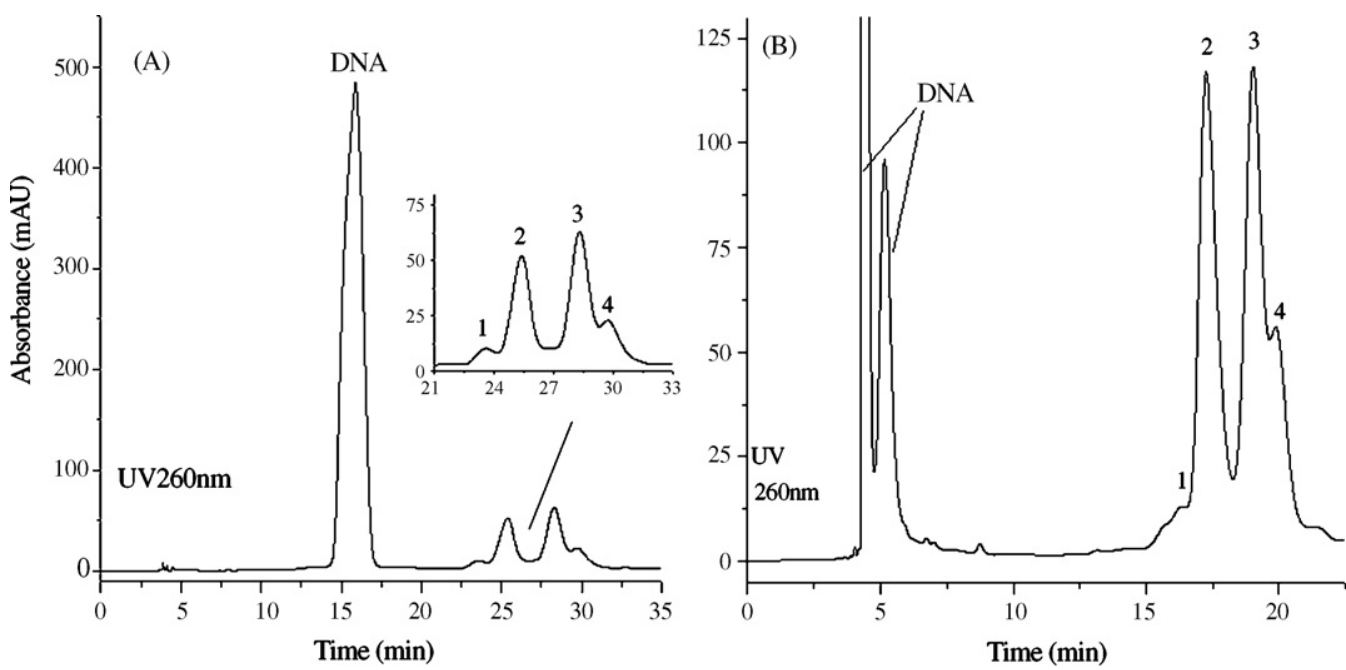

Fig. 7. HPLC preparation of four adducts using an analytical Capcell Pak C18 column (column 2, A) and an analytical Capcell Pak Ph group column (column 4, B). 

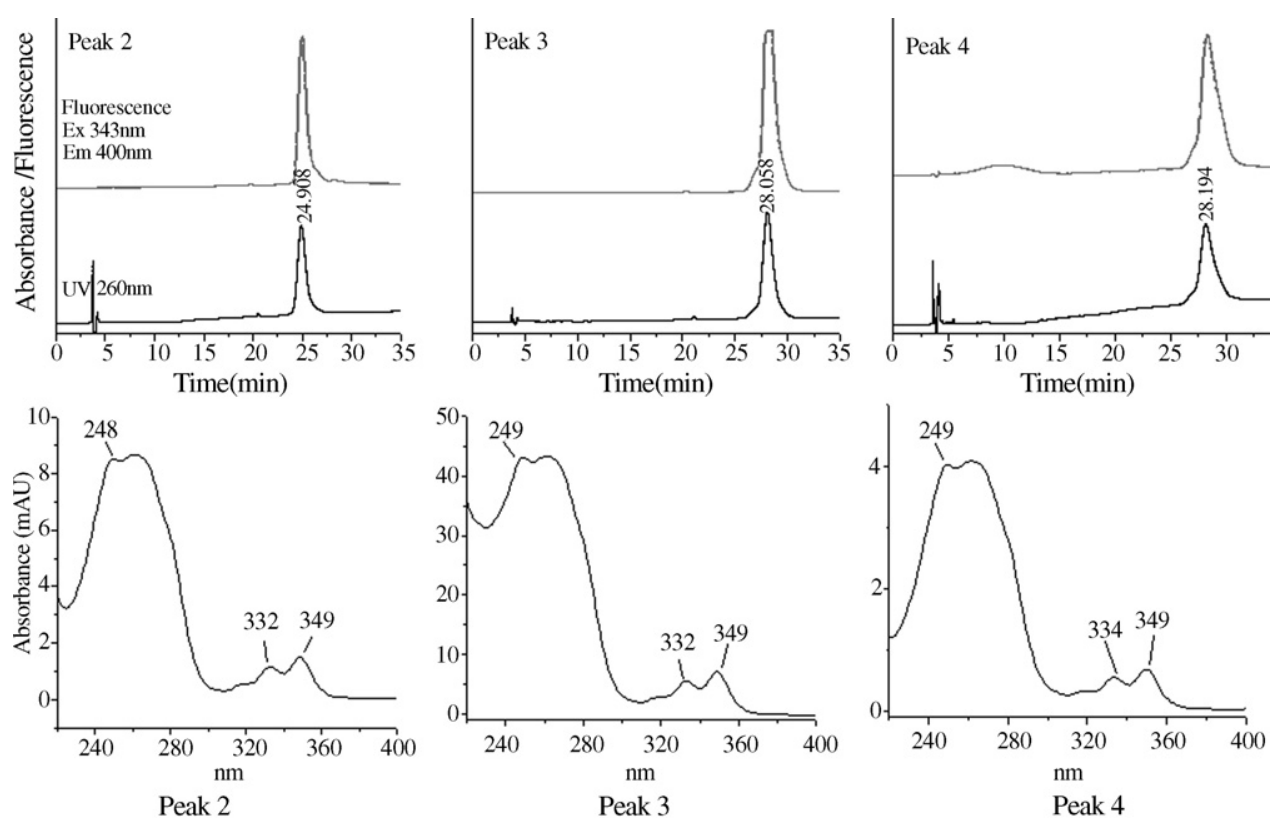

Fig. 8. HPLC analysis of the three single adducts purified by the Capcell Pak C18 column (column 2, top) and the corresponding the absorption spectra (bottom). Peaks 2, 3, and 4 are corresponding to trans-anti-BPDE-16mer A, trans-anti-BPDE-16mer B, and cis-anti-BPDE-16mer B, respectively.

from $10 \mu \mathrm{L}$ for analysis to $99 \mu \mathrm{L}$ for preparation, but the elution procedures were kept the same as described in experiment section for columns 2 and 4 , respectively. For the column 2 , it is evident that it is tolerant to this 10 times increased injection volume (Fig. 7A). Similar as the analytical separation on column 2 (Fig. 2), the well-separation of the four adduct stereoisomers for preparation on column 2 was also achieved. But for column 4, the separation of the four adduct stereoisomers became worse (Fig. 7B) in comparison with corresponding analytical separation (Fig. 6). In this work, column 2 was chosen for purification and preparation of the four adduct stereoisomers. Three adduct stereoisomers (trans-antiBPDE- $N^{2}-\mathrm{dG}-16$ mer A, trans-(+)-anti-BPDE- $N^{2}-\mathrm{dG}-16$ mer $\mathrm{B}$, and cis-anti-BPDE- $N^{2}-\mathrm{dG}-16 \mathrm{mer} \mathrm{B}$ ) were purified and prepared. The adduct of $c i$-anti-BPDE- $N^{2}-\mathrm{dG}-16$ mer A was hard to be collected due to very low concentration.

The final purified adduct products were further subjected to HPLC analysis for examination of purity and UV spectrum (Fig. 8). All three purified adduct stereoisomers (trans-antiBPDE- $N^{2}-\mathrm{dG}-16$ mer A, trans-anti-BPDE- $-N^{2}-\mathrm{dG}-16$ mer B, and cis-anti-BPDE- $N^{2}-\mathrm{dG}-16 \mathrm{mer} \mathrm{B}$, corresponding to peaks 2,3 , and 4) display as a BPDE-dG-16mer has a single adduct peak (Fig. 8). All the three adduct stereoisomers exhibit similar UV spectrum in the range from $220 \mathrm{~nm}$ to $400 \mathrm{~nm}$. Both BPDE and 16mer contribute to their UV spectra. The maximum absorbance peaks at 248/249 nm, 332/334 nm, and $349 \mathrm{~nm}$ stem from the absorbance of BPDE moiety, and the peak of $260 \mathrm{~nm}$ stem from single stranded $16 \mathrm{mer}$.

\section{Conclusions}

Here we have developed an improved HPLC method for simultaneous analysis and preparation of four stereoisomers of anti-BPDE-oligonucleotide adducts. This is achieved by opti- mizing stationary phase through reducing weak interaction of stationary phase with oligonucleotide and enhancing selective interaction of stationary phase with adduct moiety. This work provides a strategy for developing novel stationary phase for separation and analysis of aromatic DNA adducts.

\section{Acknowledgements}

This work was supported by the grants from National Natural Science Foundation of China (No.20677066 and 20621703) and National Basic Research Program of China (973 program, No.2007CB407305) to Dr. H. Wang.

\section{References}

[1] Benzo[a]pyrene, IARC monographs on the evaluation of the carcinogenic risk of chemicals to humans, International Agency for Research on Cancer (IARC), Lyon, 1983.

[2] J.M. Cook, C.L. Hewett, I. Hieger, J. Chem. Soc. 55 (1933) 395

[3] D.H. Phillips, Nature 303 (1983) 468.

[4] S.K. Yang, H.V. Gelboin, Biochem. Pharmacol. 25 (1976) 2221.

[5] S.K. Yang, D.W. McCourt, P.P. Roller, H.V. Gelboin, Proc. Natl. Acad. Sci. U.S.A. 73 (1976) 2594.

[6] D.R. Thakker, H. Yagi, W. Levin, A.Y.H. Lu, A.H. Conney, D.M. Jerina, J. Biol. Chem. 252 (1977) 6328.

[7] J. Kapitulnik, P.G. Wislocki, W. Levin, H. Yagi, D.R. Thakker, H. Akagi, M. Koreeda, M. Jerina, A.H. Conney, Cancer Res. 38 (1978) 2661.

[8] D.R. Thakker, H. Yagi, Y.H. Lu, W. Levin, A.H. Conney, D.M. Jerina, Proc. Natl. Acad. Sci. U.S.A. 73 (1976) 3381.

[9] D.R. Thakker, H. Yagi, H. Akagi, M. Koreeda, A.Y.H. Lu, W. Levin, A.W. Wood, A.H. Conney, D.M. Jerina, Chem. Biol. Interact. 16 (1977) 281.

[10] R.F. Newbold, P. Brookes, Nature 261 (1976) 52.

[11] E. Huberman, L. Sachs, S.K. Yang, H.V. Gelboin, Proc. Natl. Acad. Sci. U.S.A. 73 (1976) 607.

[12] I.C. Hsu, G.T. Bowden, C.C. Harris, Mutat. Res. 63 (1979) 351.

[13] T.J. Slaga, W.J. Bracken, G. Gleason, W. Levin, H. Yagi, D.M. Jerina, A.H. Conney, Cancer Res. 394 (1979) 67. 
[14] M.K. Buening, P.G. Wislocki, W. Levin, H. Yagi, D.R. Thakker, H. Akagi, M. Koreeda, D.M. Jerina, A.H. Conney, Proc. Natl. Acad. Sci. U.S.A. 11 (1978) 5358.

[15] A.W. Wood, R.L. Chang, W. Levin, H. Yagi, D.R. Thakker, D.M. Jerina, A.H. Conney, Biochem. Biophys. Res. Commun. 77 (1977) 1389.

[16] P. Brookes, M.R. Osborne, Carcinogenesis 3 (1982) 1223.

[17] J.A. Burgess, C.W. Stevens, W.E. Fahl, Cancer Res. 45 (1985) 4257.
[18] S. Shibutani, L.A. Margulis, N.E. Geacintov, A.P. Grollman, Biochemistry 32 (1993) 7531.

[19] S.C. Cheng, B.D. Hilton, J.M. Roman, A. Dipple, Chem. Res. Toxicol. 2 (1989) 334.

[20] T.J. Carnelley, S. Barker, H.L. Wang, W.G. Tan, M. Weinfeld, X.C. Le, Chem. Res. Toxicol. 14 (2001) 1513.

[21] M. Funk, I. Ponten, A. Seidel, B. Jernstrom, Bioconjugate Chem. 8 (1997) 310. 